\title{
Study Hydrophobicity and Antigenicity of Cytochrome C Oxidase Subunit II from D. medinensis: New Prototype of Synthetic Vaccine Development
}

Sonu Mishra and Virendra S Gomase*

Department of Biotechnology, Mewar University, Chittorgarh, India

\begin{abstract}
Cytochrome $\mathrm{c}$ oxidase subunit II, also known as cytochrome $\mathrm{c}$ oxidase polypeptide II which is an oligomeric enzyme. In this study Cytochrome c oxidase subunit 2 (mitochondrion) protein has been used to investigate its role in antigenicity. Cytochrome c oxidase subunit 2 protein sequences ( 230 aa protein) is analyzed through different types B- cell epitope prediction methods. We found that the region of maximal hydrophilicity is likely to be an antigenic site, having hydrophobic characteristics, because the terminal regions of antigen protein is solvent accessible and unstructured, antibodies against those regions are also likely to recognize the native protein. It was seen that an antigen protein is hydrophobic in nature and contains segments of low complexity and high-predicted flexibility. The predicted antigenic protein segments of Cytochrome c oxidase subunit 2 can take active part in the host immune reactions. In future study the predicted antigenic protein Cytochrome $\mathrm{c}$ oxidase subunit 2 fragments can be used in the investigation of MHC molecules binding and it can be the first bottlenecks in vaccine design.
\end{abstract}

Keywords: Antigen; Dracunculus medinensis; Epitope; Protein; Synthetic vaccine; Cytochrome c oxidase subunit II (mitochondrion)

\section{Introduction}

Dracunculiasis, is caused by a $60-100 \mathrm{~cm}$ long nematode worm, Dracunculus medinensis, via a drinking of contaminated water infected with copepod Cyclops (intermediate host). Dracunculiasis has been known to humankind since antiquity. Guinea worm the largest tissue parasite with unusual life cycle with incubation period of the approximately more than a year with six developmental stages. This one of the most neglected tropic parasite which bears clinical importance and needs to be eradicated after small pox [1]. Mature and adult female after the copulation produces millions of eggs in its uterus, and is predominantly localized in the lower extremities (80-90\%). After an incubation period the female worm release the larvae which induces a painful blister (1 to $6 \mathrm{~cm}$ diameter) on the skin of lower limbs; the person develop a slight fever, local skin redness, swelling and severe pruritus around the blister. Other symptoms include diarrhoea, nausea, vomiting and dizziness. The severity of the wound infections in the infected individual led to a more complications such as redness and swelling of the skin (cellulitis), boils (abscesses), generalized infection (sepsis), joint infections (septic arthritis) that can cause the joints to lock and deform (contractures), lock jaw (tetanus). The blister burst within 1 to 3 days and female worms one or more slowly comes out from the wounds which causes an excoriating burning sensation and pain $[2,3]$. Immersing or pouring water over the blister provides pain relief. But this the moment that adult female is exposed to the external environment [4]. During emergence of the limbs in open water sources it recognizes the temperature difference and releases the milky white liquid in the water which contains millions of immature larvae, when larvae released in water are ingested by copepods where they mount twice and become infective larvae within two weeks [5].

The $D$. medinensis antigen peptides can be most desirable segment for the subunit vaccine development because with the single epitope, the immune response can be generated in large population. This approach is usually based on the phenomenon of cross-protection, whereby infected with the mild strain and is protected against a more severe strain of the same. The phenotype of the resistant transgenic hosts includes fewer centers of initial infection, a delay in symptom development and low accumulation. In this study cytochrome c oxidase subunit II (mitochondrion) protein has been used to investigate its role in antigenicity. Cytochrome c oxidase subunit 2, also known as cytochrome c oxidase polypeptide II which is an oligomeric enzyme, an important component of the respiratory chain which involves in the transfer of electrons from cytochrome $c$ to oxygen. This enzyme complex is found located in the mitochondrial inner membrane in eukaryotes. Cytochrome $\mathrm{c}$ oxidase subunit 2 contains two adjacent transmembrane regions in its $\mathrm{N}$-terminus. The considerable part of the protein is generally exposed to the periplasmic or to the mitochondrial intermembrane space. The $\mathrm{N}$-terminal domain of cytochrome $\mathrm{C}$ oxidase contains two transmembrane alpha-helices. Cytochrome oxidase deficiency and abnormality has been seen in the Leigh's disease. Investigation shows that any alterations in the catalytic genes of cytochrome c oxidase subunits I and II (COI and COII) have an adverse impact on prognosis in patients with acute myeloid leukaemia (AML) [6,7]. A "mitochondrial hypothesis" of late onset Alzheimer's disease $(\mathrm{AD})$ has been proposed. The in depth biochemical studies propose that there is a significant decrease in cytochrome oxidase (CO) activity as well as perturbed CO I and CO III mRNA levels in platelets and brain tissue from Alzheimer's patients. The phenotypic expression study of the $\mathrm{CO}$ mutation is the major reason for reduced $\mathrm{CO}$ activity and compromised mitochondrial function [8]. Antigen protein prediction from $D$. medinensis is necessary for few paradigms of synthetic vaccine development and target validation.

\section{Methodology}

B-cell epitopes are the sites of molecules that are recognized by antibodies of the immune system. Knowledge of B-cell epitopes may

*Corresponding author: Virendra S. Gomase, Department of Biotechnology, Mewar University, Chittorgarh, India, Tel: +9127743967; E-mail: gomase.viren@gmail.com

Received December 11, 2015; Accepted January 07, 2016; Published January 12,2016

Citation: Mishra S, Gomase VS (2016) Study Hydrophobicity and Antigenicity of Cytochrome C Oxidase Subunit II from D. medinensis: New Prototype of Synthetic Vaccine Development. Immunochem Immunopathol 2: 113. doi:10.4172/2469 9756.1000113

Copyright: ( 2016 Mishra S, et al. This is an open-access article distributed under the terms of the Creative Commons Attribution License, which permits unrestricted use, distribution, and reproduction in any medium, provided the original author and source are credited. 
be used in the design of vaccines and diagnostics tests. It is therefore of interest to develop improved methods for predicting B-cell epitopes [9]. In this research work, antigenic epitopes of antigen protein cytochrome c oxidase subunit II (mitochondrion) from D. medinensis is determined using the Gomase in 2007, Bepipred Linear Epitope Prediction, Emini Surface Accessibility Prediction, Karplus and Schulz Flexibility Prediction, Kolaskar and Tongaonkar Antigenicity, Parker Hydrophilicity Prediction [10-18].

\section{Database searching}

The antigenic protein sequence of Dracunculus medinensis Antigen cytochrome c oxidase subunit II was retrieved from www.ncbi.nlm.nih. gov, UniProt databases are initially the most important $[19,20]$.

\section{Prediction of antigenicity}

Prediction of antigenicity program predicts those segments from cytochrome coxidase subunit II protein that are likely to be antigenic by eliciting an antibody response. In this research work antigenic epitopes of cytochrome c oxidase subunit II from Dracunculus medinensis are determined by using the Hopp and Woods, Welling, Parker, Bepipred, Kolaskar and Tongaonkar antigenicity methods [21-24].

\section{Solvent accessible regions}

We also predict solvent accessible regions of proteins having highest probability that a given protein region lies on the surface of a protein Surface Accessibility, backbone or chain flexibility by Emini et al. [25] and Karplus and Schulz [26]. By using different scale we predict the hydrophobic and hydrophilic characteristics of amino acids that are rich in charged and polar residues i.e., Sweet et al. [27], Kyte and Doolittle [28], Abraham and Leo [29], Bull and Breese [30], Miyazawa et al. [31], Roseman [32], Wilson et al. [33], Cowan [34].

\section{Results and Interpretation}

The cytochrome c oxidase subunit II from Dracunculus medinensis, contain a long residue of 230 amino acids.

MGSFFHGFNFNFMVSHLSSGMDWFHCFGCSFLLMILVFV VFLFYNLMGSRYYYKSFEDDYRFIEFFCSIFPSLVLLILM VPSLSLLYEYSMMNFSSDLNVGVVGHQWYWSYEYSDYT DEVGFDSYMLPSEDMILGDLRLLDVDNRCVIPSGVSVGFLIGSEDVIHSWALPCMSIKVDAVGGAISRVTCVFPLIGLYYGQCSEICGAYHSFMPIVIESTISENFVKWVAGS

\section{Prediction of antigenic peptides}

In this study, we found the antigenic determinants by finding the area of greatest local hydrophilicity (Figure 1). The HoppWoods scale Hydrophilicity Prediction Result Data found high in position between 55-60 (55-SFEDDY-60) in a protein, assuming that the antigenic determinants would be exposed on the surface of the protein and thus would be located in hydrophilic regions (Figure 2). Welling antigenicity plot gives value as the log of the quotient between percentage in a sample of known antigenic regions and percentage in average proteins and Prediction Result Data found high in position 142-146 (142- VDNRC-146) (Figure 3). We also study Hydrophobicity plot of HPLC / Parker Hydrophilicity Prediction Result Data found between 111-115 (Maximum Score 5.1) i.e., the maximum predicted residues at the position 115(Residue is D) is 112- EYSDYTD-118 and at position 116(Residue is $\mathrm{Y}$ ) with start and end position is 113YSDYTDE-119 (Figure 4), BepiPred predicts the location of linear

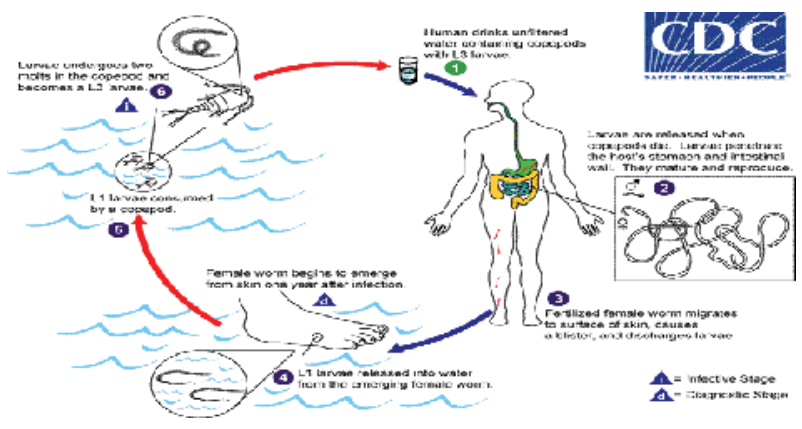

Figure 1: Guinea worm life cycle and interventions to interrupt transmission [6].

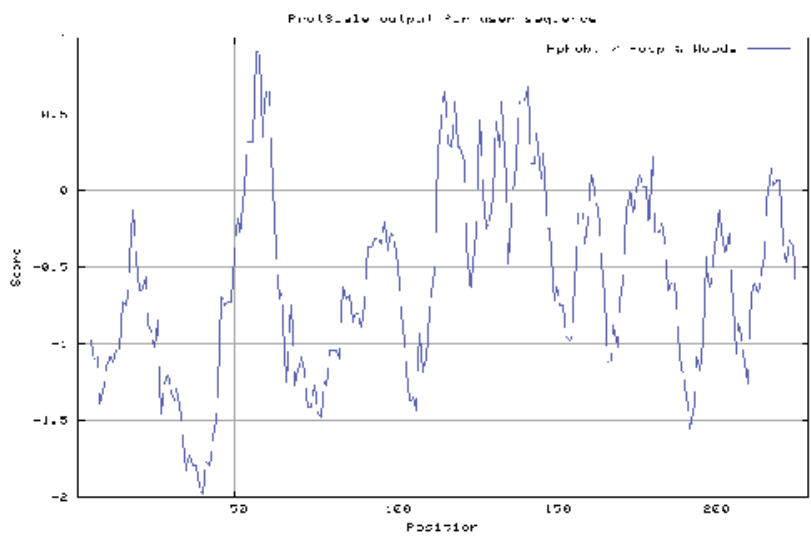

Figure 2: Hydrophobicity plot of Hopp and Woods (1981) of cytochrome c oxidase subunit II.

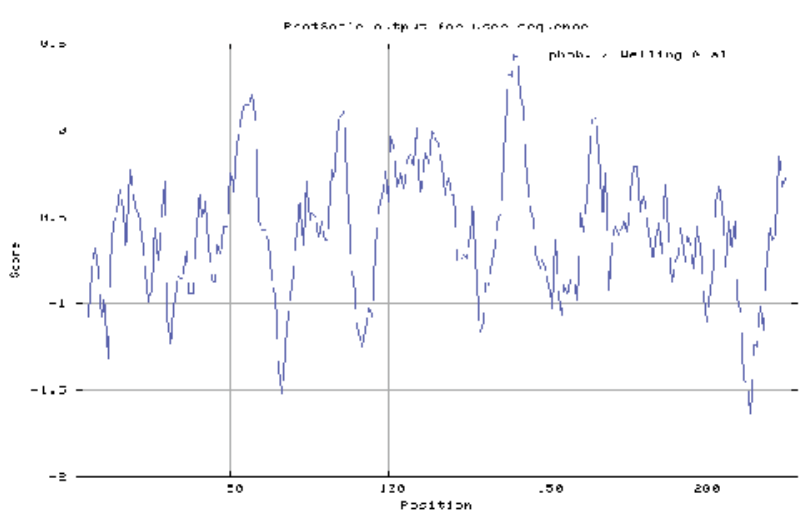

Figure 3: Hydrophobicity plot of Welling et al. (1985) of cytochrome c oxidase subunit II.

B-cell epitopes Result found at position 115, the residue is D and the maximum score is 1.101 (Figure 5), Kolaskar and Tongaonkar antigenicity methods (Figure 6) Predicted peptides result found i.e., 22-DWFHCFGCSFLLMILVFVVFLFY-44, 48-GSRYYYK-54,61RFIEFFCSIFPSLVLLILMVPSLSLLYE-88, 96-SDLNVGVVGH-105, 121-GFDSYML-127,133-ILGDLRLLDVDNRCVIPSGVSVGFLIGSE DVIHSWALPCMSIKVDAVGGAISRVTCVFPLIGLYYGQCSEICG AYHSFMPIVIE-216 (Table 1). The average antigenic propensity for protein found is 1.0660 and the predicted antigenic fragments can bind to MHC molecule is the first bottlenecks in vaccine design. 


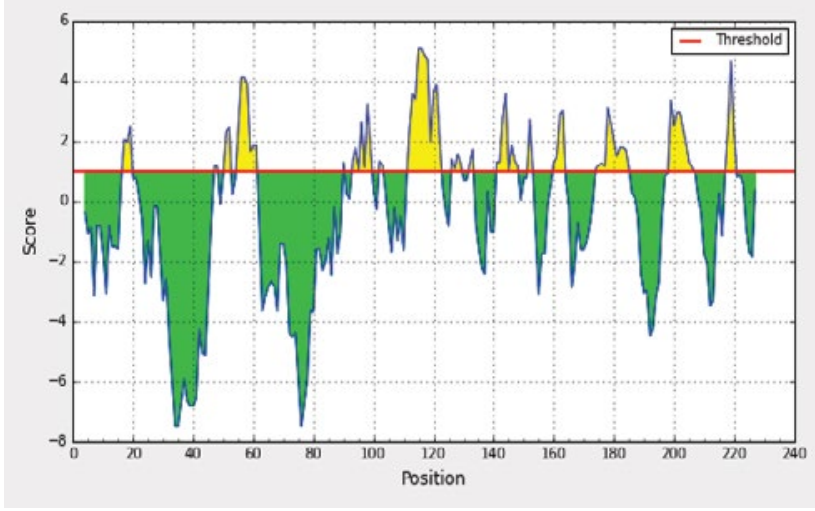

Figure 4: Hydrophobicity plot of HPLC/Parker et al. (1986) of cytochrome c oxidase subunit II.

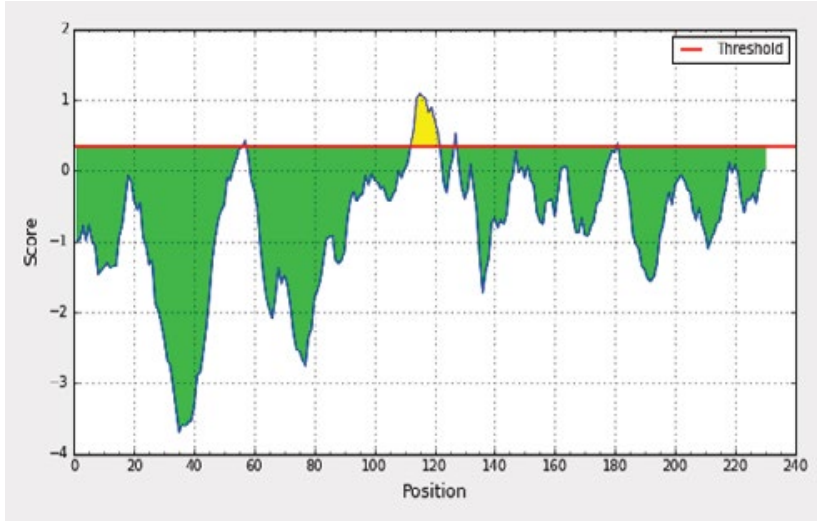

Figure 5: Bepipred Linear Epitope Prediction Graph of cytochrome c oxidase subunit II.

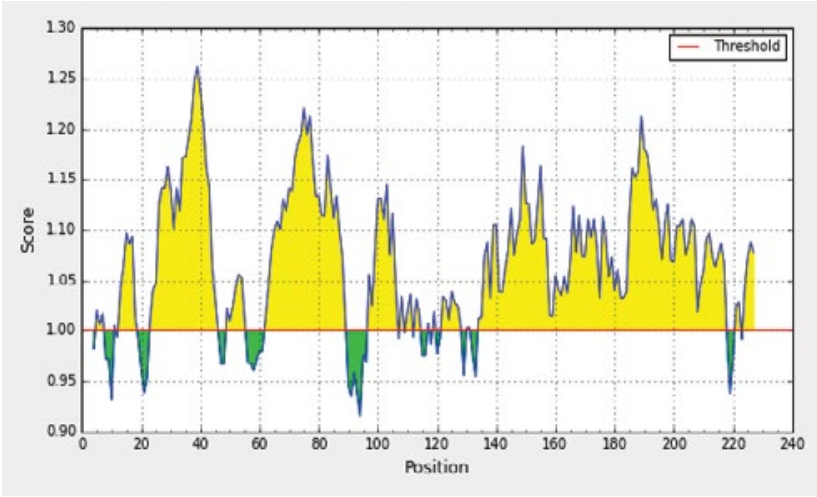

Figure 6: Kolaskar and Tongaonkar Antigenicity Prediction Graph of cytochrome c oxidase subunit II.

\section{Solvent accessible regions}

We also predict solvent accessible regions in proteins; different measurement was performed for the prediction of antigenic activity, surface region of peptides. Emini et al. (Figure 7) predicts the highest probability i.e., found at position 51(Residue is Y) with start and end position is 49- SRYYYK-54(Maximum score 7.499) and at position 52 (Residue Y) with start and end position is 50- RYYYKS-55(Maximum score 7.499), that a given protein region lies on the surface of a protein and are used to identify antigenic determinants on the surface of proteins. Karplus and Schulz (Figure 8) high score (1.061) is found at position 129(Residue S) with start and the end position 126MLPSEDM-132. Predict backbone or chain flexibility on the basis of the known temperature B factors of the a-carbons. The hydrophobic and hydrophilic characteristics of amino acids is determined by using different scales that are rich in charged and polar residues i.e., Sweet et al. hydrophobicity prediction Result Data found high in position 3543 i.e., 35-ILVFVV FLFYN-43 (Figure 9), Kyte and Doolittle result high in position 36 with maximum score 3.689 (Figure 10), Abraham and Leo result high in position: 39, Score: $2.243(\max )$ (Figure 11), Bull and Breese result high in position: 202, Score: $0.378(\max )$ (Figure 12), Miyazawa result high in position: 35, Score: $8.501(\max )$ (Figure 13), Roseman result high in position: 39, Score: 1.797 (Figure 14), Wilson et result high in position: 40, Score: 6.722 (Figure 15), Cowan result high in position: 39 , score: 1.628 (Figure 16), Rose et al. result high in Position: 39, Score: $0.867(\max )$ (Figure 17), Eisenberg et al. result high in Position: 39, Score: $1.146(\max )(\max )$ (Figure 18), Rao and Agros results high at position: 39, Score: 1.467 (max) (Figure 19), Manavalan et al. result high at position: 36, Score: $15.099(\max )$ (Figure 20). In this study, we found the antigenic determinants by finding the area of greatest local hydrophilicity. Hopp and Woods hydrophobicity scale is used to identify of potentially antigenic sites in proteins by analyzing amino acid sequences in order to find the point of greatest hydrophilic. Hydrophilicity Prediction result data found high in sequence position at 55-60 (55-SFEDDY-60) in a protein this scale is basically a hydrophilic index where apolar residues have been assigned negative values. The Window size of 5-7 is good for finding hydrophilic regions, greater than 0 values are consider as hydrophilic which is consider as antigenic. Welling used information on the relative occurrence of amino acids in antigenic regions to make a scale which is useful for prediction of antigenic regions and the predicted result data found high in sequence position 142-146 (142- VDNRC-146). Welling antigenicity plot gives value as the log of the quotient between percentage in a sample of known antigenic regions and percentage in average proteins. We also study Hydrophobicity plot of HPLC / Parker Hydrophilicity Prediction Result Data found between 111-115 (Maximum Score 5.1) i.e., the maximum predicted residues at the position 115 (Residue is $\mathrm{D}$ ) is 112- EYSDYTD-118 and at position 116 (Residue is Y) with start and end position is 113- YSDYTDE-119 (Table 2). BepiPred predicts the location of linear B-cell epitopes Result found in position 115, the residue is $\mathrm{D}$ and the maximum score is 1.101 (Table 3). There are 6 antigenic determinant sequences is found by Kolaskar and Tongaonkar antigenicity scales the results show highest pick at position Predicted peptides result found i.e., 22-DWFHCFGCSFLLMILVFVVFLFY-44, 48-GSRYYYK-54, 61-RFIEFFCSIFPSLVLLILMVPSLSLLYE-88, 96-SDLNVGVVGH-105， 121-GFDSYML-127,133-ILGDLRLLDVD NRCVIPSGVSVGFLIGSEDVIHSWALPCMSIKVDAVGGAISRVTC VFPLIGLYYGQCSEICGAYHSFMPIVIE-216. Result of determined antigenic sites on proteins has revealed that the hydrophobic residues if they occur on the surface of a protein are more likely to be a part of antigenic sites. This method can predict antigenic determinants with about $75 \%$ accuracy and also gives the information of surface accessibility and flexibility. Further this region form beta sheet which show high antigenic response than helical region of this peptide and shows highly antigenicity. We predict Solvent accessibility by using Emani et al. the result found the highest probability i.e., found at position 51(Residue is $\mathrm{Y}$ ) with start and end position is 49- SRYYYK-54(Maximum score 7.499) and at position 52 (Residue $Y$ ) with start and end position is 50RYYYKS-55(Maximum score 7.499), that a given protein region lies on 


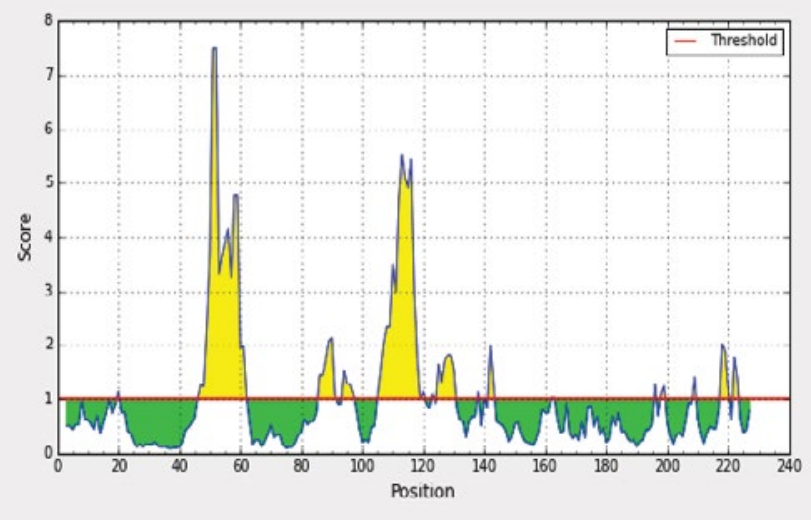

Figure 7: Emini Surface Accessibility Prediction Graph of cytochrome c oxidase subunit II.

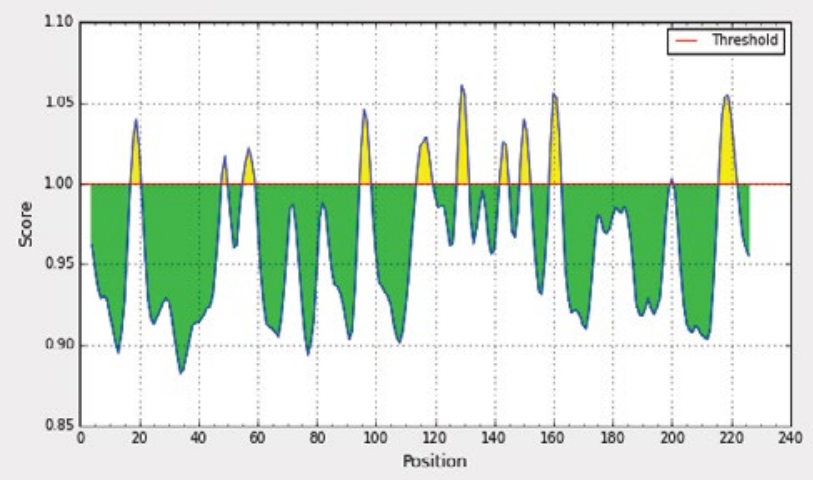

Figure 8: Karplus and Schulz Flexibility Prediction Graph of cytochrome c oxidase subunit II.

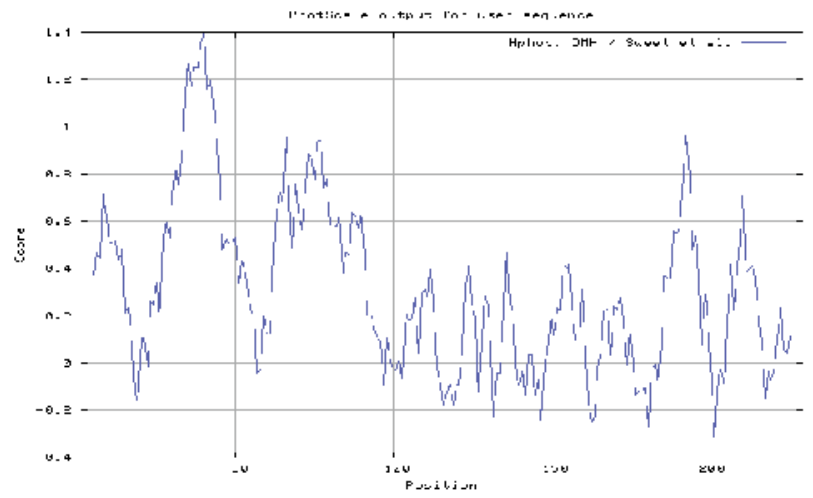

Figure 9: Hydrophobicity plot of Sweet et al. (1983) of cytochrome c oxidase subunit II.

the surface of a protein and are used to identify antigenic determinants on the surface of proteins. This algorithm also used to identify the antigenic determinants on the surface of proteins and Karplus and Schulz predict backbone or chain flexibility on the basis of the known temperature B factors of the a-carbons here we found the result with high score (1.061) is found at position 129 (Residue S) with start and the end position 126- MLPSEDM-132. We predict Solvent accessibility of cytochrome c oxidase subunit II for delineating hydrophobic and

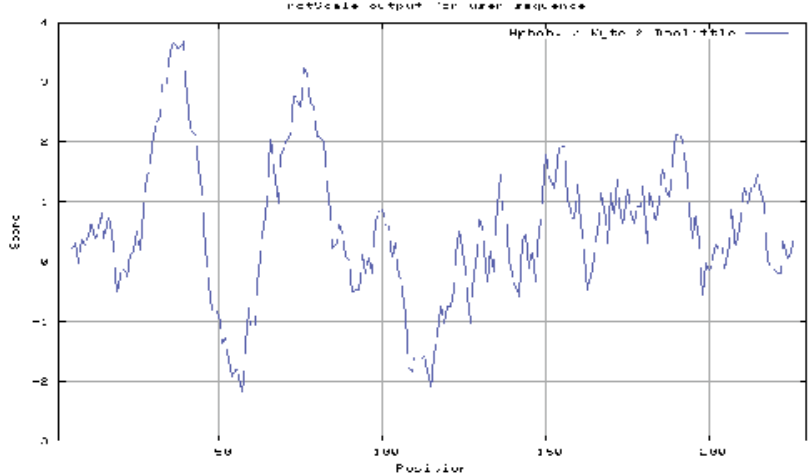

Figure 10: Kyte and Doolittle hydrophobicity plot of cytochrome c oxidase subunit II.

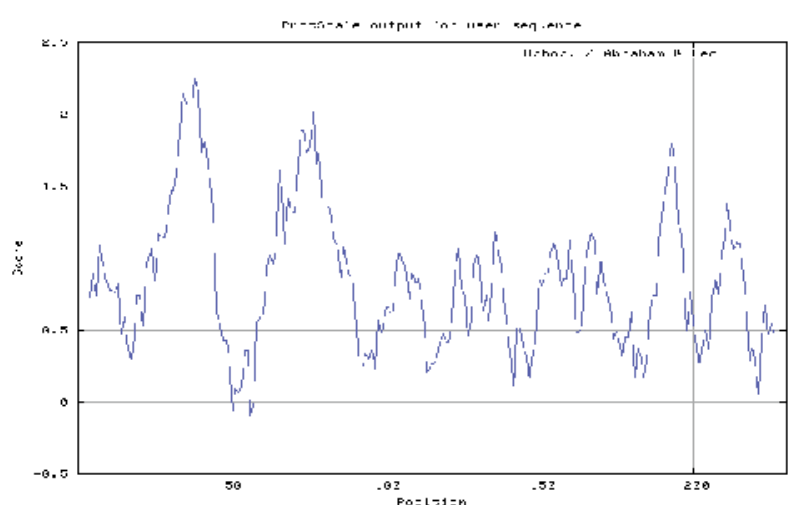

Figure 11: Abraham and Leo hydrophobicity plot of cytochrome c oxidase subunit II.

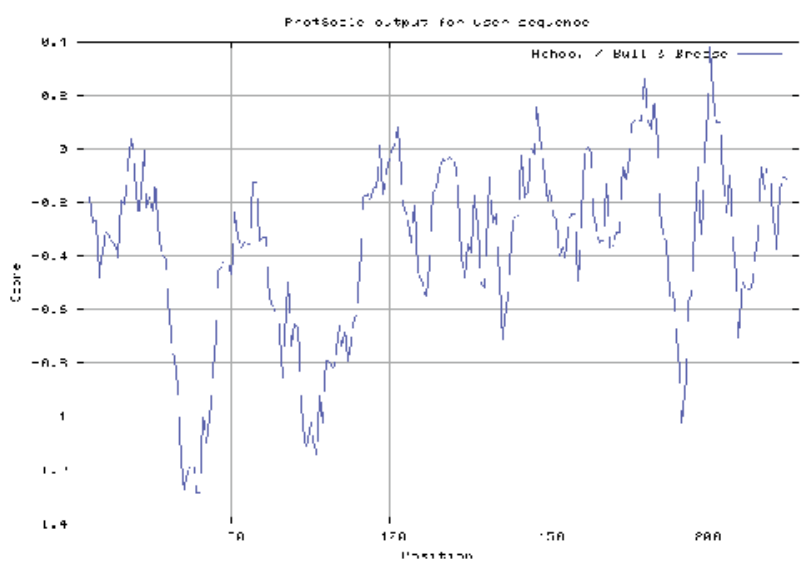

Figure 12: Bull and Breese use surface tension to measure hydrophobicity and also uses negative values to describe the hydrophobicity of cytochrome c oxidase subunit II.

hydrophilic characteristics of amino acids. Solvent accessibility used to identify active site of functionally important residues in membrane proteins. Solvent-accessible surface areas and backbone angles are continuously varying because proteins can move freely in a threedimensional space. The mobility of protein segments which are located on the surface of a protein due to an entropic energy potential and 


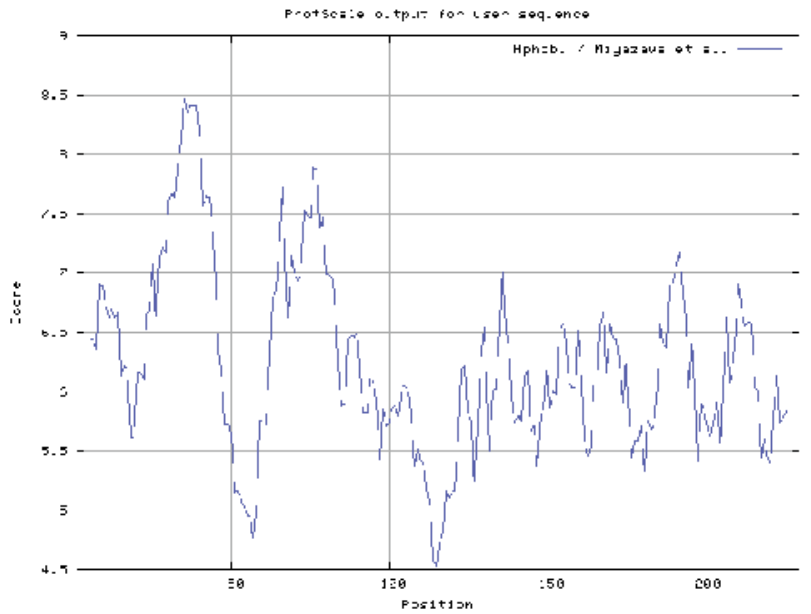

Figure 13: Hydrophobicity plot of Miyazawa et al. (1985) of cytochrome c oxidase subunit II.

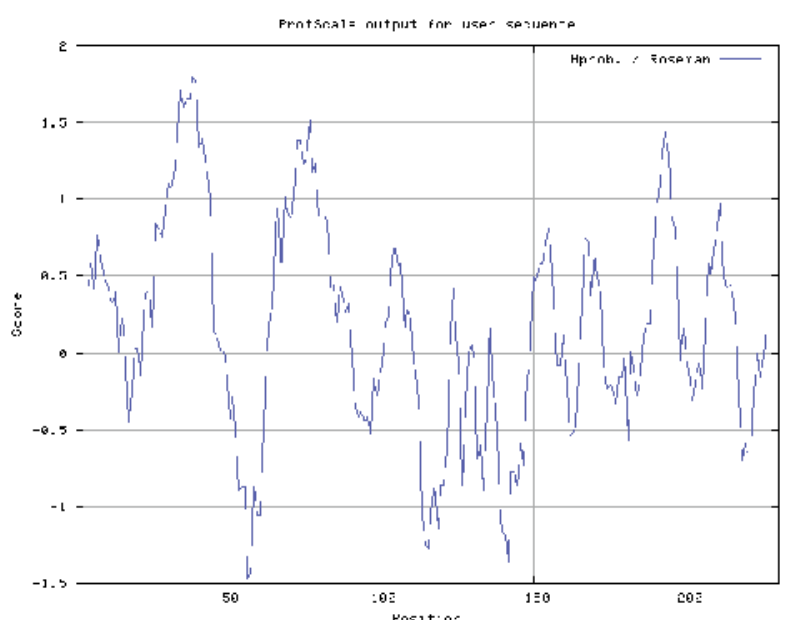

Figure 14: Hydrophobicity plot of Roseman MA (1988) of cytochrome c oxidase subunit II.

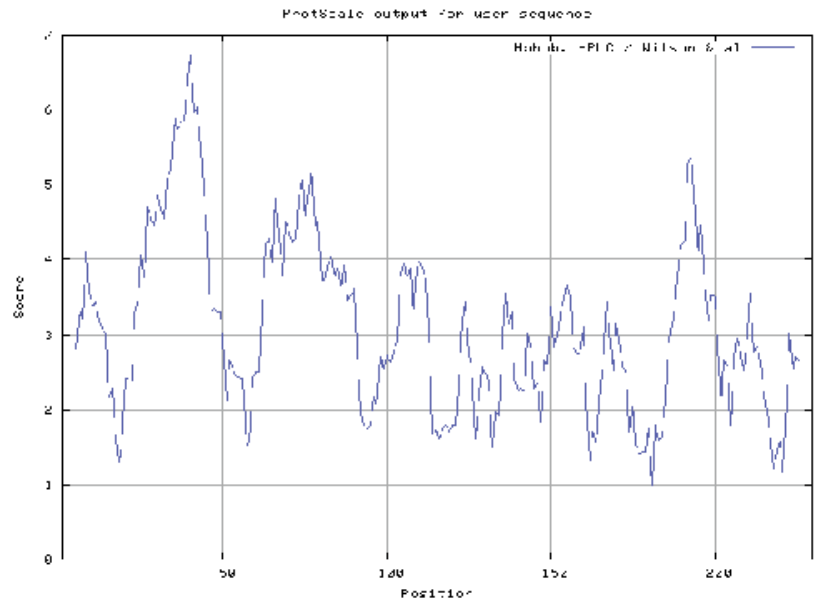

Figure 15: Hydrophobicity/HPLC plot of Wilson et al. (1981) of cytochrome C oxidase subunit II.

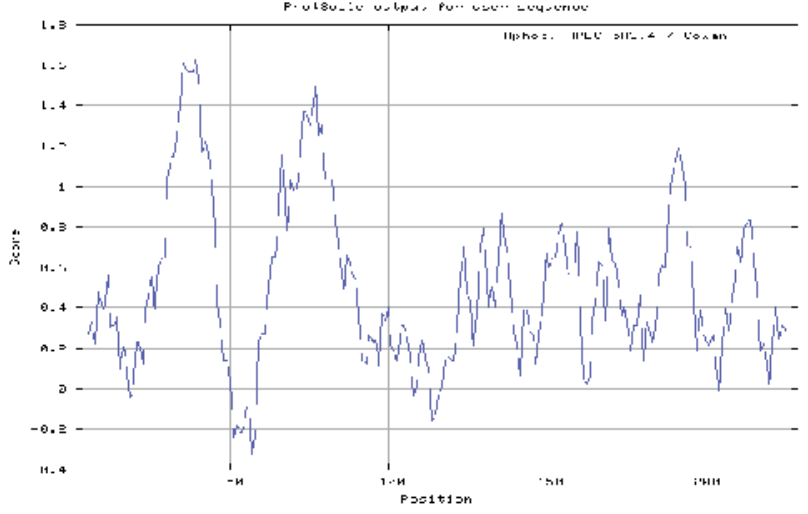

Figure 16: Hydrophobicity/HPLC pH 3.4/ plot of Cowan (1990) of cytochrome c oxidase subunit II.

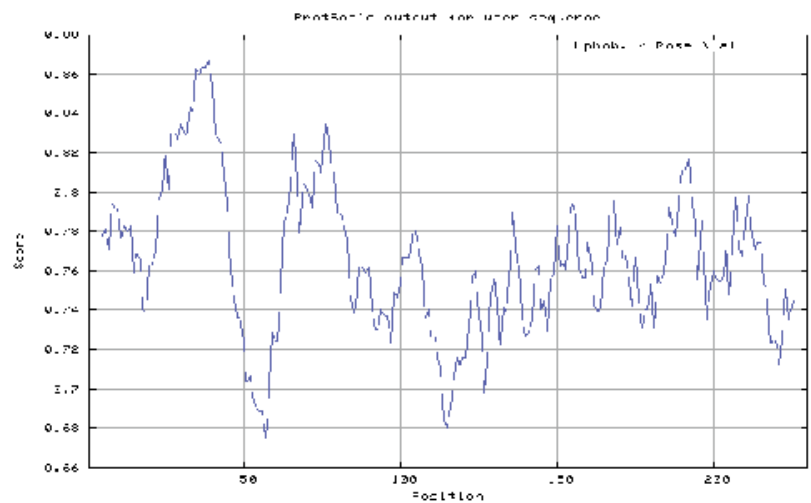

Figure 17: Hydrophobicity plot of antigen by Hphob/ Rose et al. scale of cytochrome c oxidase subunit II.

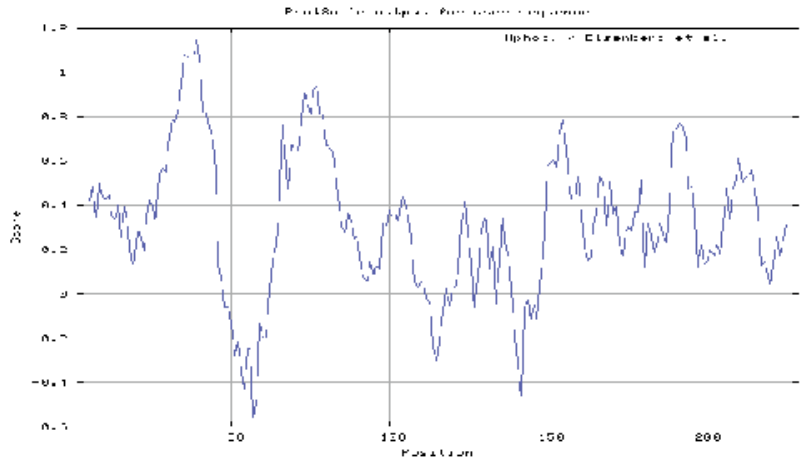

Figure 18: Hydrophobicity plot of antigen by Hphob/Eisenberg et al. scale of cytochrome c oxidase subunit II.

which seem to correlate well with known antigenic determinants. We also found the i.e., Sweet et al. hydrophobicity prediction Result Data found high in position 3543 i.e., 35-ILVFVV FLFYN-43, Kyte and Doolittle result high in position 36 with maximum score 3.689 , Abraham and Leo result high in position:39, Score: 2.243 (max), Bull and Breese result high in position: 202, Score: $0.378(\max )$, Miyazawa result high in position: 35, Score: $8.501(\max )$, Roseman result high in position:39, Score: 1.797, Wilson et result high in position:40, score: 


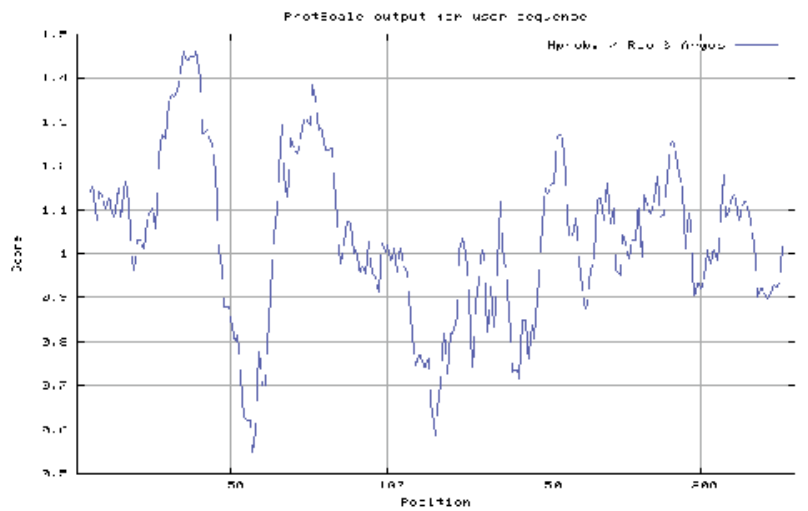

Figure 19: Antigenicity plot of antigen protein by Hphob./Rao and Argos, scale of cytochrome c oxidase subunit II.

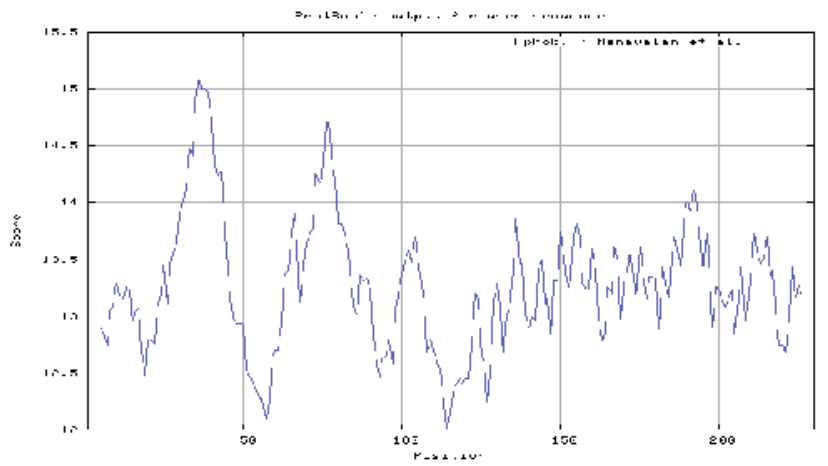

Figure 20: Antigenicity plot of antigen protein by Hphob./Manavalan et al. scale of cytochrome c oxidase subunit II.
6.722, Cowan result high in position:39, Score: 1.628 Rose et al. result high in Position: 39, Score: $0.867(\max )$, Eisenberg et al. result high in Position: 39, Score: $1.146(\max )(\max )$, Rao and Agros results high at position: 39, Score: 1.467 (max), Manavalan et al. result high at position: 36, Score: 15.099 ( $\max$ ). These scales are a hydrophilic with a polar residues assigned negative value. Because the $\mathrm{N}$ - and $\mathrm{C}$ - terminal regions of proteins are usually solvent accessible and unstructured, antibodies against those regions recognize the antigenic protein. We found that the region of maximal Hydrophilicity is likely to be an antigenic site, having hydrophobic characteristics, because the terminal regions of antigen protein is solvent accessible and unstructured, antibodies against those regions are also likely to recognize the native protein. It was seen that an antigen protein is hydrophobic in nature and contains segments of low complexity and high-predicted flexibility. The predicted antigenic protein segments of cytochrome $c$ oxidase subunit II (mitochondrion) can take active part in the host immune reactions. In future study the predicted antigenic protein cytochrome $\mathrm{c}$ oxidase subunit II (mitochondrion) fragments can be used in the investigation of MHC molecules binding via utilizing the bioinformatics tools and software, and it can be the first bottlenecks in vaccine design.

\section{Conclusion}

An antigenic protein can plays an important role in vaccine development. The peptide fragments of antigen protein can be used to select naonamer for use in rational vaccine design and can develop the understanding of roles in the immune system in infectious disease. Overall, the results are encouraging, both the 'sites of action' and 'physiological functions' can be predicted with very high accuracies helping minimize the number of validation experiments. From the above result and discussion it is concluded that an antigenic protein cytochrome $\mathrm{c}$ oxidase subunit II from $D$. medinensis can play an important role in the vaccine development.

\begin{tabular}{|c|c|c|c|}
\hline \multicolumn{4}{|c|}{ There are 6 antigenic determinants in cytochrome $c$ oxidase subunit II protein sequence } \\
\hline $\mathbf{n}$ & Start Position & Sequence & End Position \\
\hline 1 & 22 & DWFHCFGCSFLLMILVFVVFLFY & 44 \\
\hline 2 & 48 & GSRYYYK & 54 \\
\hline 3 & 61 & RFIEFFCSIFPSLVLLILMVPSLSLLYE & 88 \\
\hline 4 & 96 & SDLNVGVVGH & 105 \\
\hline 5 & 121 & GFDSYML & 127 \\
\hline 6 & 133 & $\begin{array}{l}\text { ILGDLRLLDVDNRCVIPSGVSVGFLIGSEDVIHSWALPCMSIKV } \\
\text { DAVGGAISRVTCVFPLIGLYYGQCSEICGAYHSFMPIVIE }\end{array}$ & 216 \\
\hline
\end{tabular}

Table 1: Kolaskar and Tongaonkar Antigenicity Prediction Graph of cytochrome c oxidase subunit II.

\begin{tabular}{|c|c|c|c|c|c|c|}
\hline \multirow{12}{*}{$\begin{array}{c}\text { Parker Hydrophilicity } \\
\text { Prediction-Predicted residue } \\
\text { scores }\end{array}$} & Position & Residue & Start & End & Peptide & Score \\
\hline & 110 & $\mathrm{~S}$ & 107 & 113 & WYWSYEY & -1.629 \\
\hline & 111 & $Y$ & 108 & 114 & YWSYEYS & 0.729 \\
\hline & 112 & $E$ & 109 & 115 & WSYEYSD & 2.429 \\
\hline & 113 & $Y$ & 110 & 116 & SYEYSDY & 3.586 \\
\hline & 114 & $S$ & 111 & 117 & YEYSDYT & 3.4 \\
\hline & 115 & $\mathrm{D}$ & 112 & 118 & EYSDYTD & 5.1 \\
\hline & 116 & $Y$ & 113 & 119 & YSDYTDE & 5.1 \\
\hline & 117 & $\mathrm{~T}$ & 114 & 120 & SDYTDEV & 4.843 \\
\hline & 118 & $\mathrm{D}$ & 115 & 121 & DYTDEVG & 4.729 \\
\hline & 119 & $E$ & 116 & 122 & YTDEVGF & 1.986 \\
\hline & 120 & V & 117 & 123 & TDEVGFD & 3.686 \\
\hline
\end{tabular}

Table 2: Parker Hydrophilicity Prediction-Predicted Residue Scores Table. 
Citation: Mishra S, Gomase VS (2016) Study Hydrophobicity and Antigenicity of Cytochrome C Oxidase Subunit II from D. medinensis: New Prototype of Synthetic Vaccine Development. Immunochem Immunopathol 2: 113. doi:10.4172/2469-9756.1000113

Page 7 of 7

\begin{tabular}{|c|c|c|c|c|}
\hline \multirow{12}{*}{$\begin{array}{c}\text { Bepipred Linear Epitope } \\
\text { Prediction-Predicted residue } \\
\text { scores }\end{array}$} & Position & Residue & Score & Assignment \\
\hline & 110 & $S$ & 0.02 & - \\
\hline & 111 & $Y$ & 0.108 & - \\
\hline & 112 & $E$ & 0.358 & $E$ \\
\hline & 113 & Y & 0.575 & $E$ \\
\hline & 114 & $\mathrm{~S}$ & 1.014 & $E$ \\
\hline & 115 & D & 1.101 & $E$ \\
\hline & 116 & $Y$ & 1.06 & $E$ \\
\hline & 117 & $\mathrm{~T}$ & 1.009 & $E$ \\
\hline & 118 & $\mathrm{D}$ & 0.829 & $E$ \\
\hline & 119 & $E$ & 0.893 & $E$ \\
\hline & 120 & V & 0.772 & $E$ \\
\hline
\end{tabular}

Table 3: Bepipred Linear Epitope Prediction -Predicted Residue Scores Table.

\section{Conflicts of Interest}

The authors declare no conflict of interest.

\section{References}

1. Greenaway C (2004) Dracunculiasis (guinea worm disease). CMAJ 170: 495500 .

2. Muller R (1971) Dracunculus and dracunculiasis. Adv Parasitol 9: 73-151.

3. Muller R (1979) Guinea worm disease: epidemiology, control, and treatment. Bull World Health Organ 57: 683-689.

4. Ruiz-Tiben E, Hopkins DR (2006) Dracunculiasis (Guinea worm disease) eradication. Adv Parasitol 61: 275-309.

5. Iriemenam NC, Oyibo WA, Fagbenro-Beyioku AF (2008) Dracunculiasis--the saddle is virtually ended. Parasitol Res 102: 343-347.

6. (2015) Centers for Disease Control and Prevention. Parasites - Dracunculiasis (also known as Guinea Worm Disease).

7. Silkjaer T, Nyvold CG, Juhl-Christensen C, Hokland P, Nørgaard JM (2013) Mitochondrial cytochrome $\mathrm{c}$ oxidase subunit II variations predict adverse prognosis in cytogenetically normal acute myeloid leukaemia. Eur J Haematol 91: 295-303

8. Hamblet NS, Ragland B, Ali M, Conyers B, Castora FJ (2006) Mutations in mitochondrial-encoded cytochrome $c$ oxidase subunits I, II, and III genes detected in Alzheimer's disease using single-strand conformation polymorphism. Electrophoresis 27: 398-408.

9. Larsen JE, Lund O, Nielsen M (2006) Improved method for predicting linear B-cell epitopes. Immunome Res 2: 2.

10. Gomase VS, Kale KV, Chikhale NJ, Changbhale SS (2007) Prediction of MHC binding peptides and epitopes from alfalfa mosaic virus. Curr Drug Discov Technol 4: 117-215.

11. Gomase VS, Kale KV (2008) In silico prediction of epitopes: a new approach for fragment based viral peptide vaccines. Int. J. of Applied Computing 1: 39-46.

12. Gomase VS, Kale KV (2008) Approach of proteomics system architecture in plant virus's database. Int J of Applied Computing 1: 33-38.

13. Gomase VS, Chitlange NR (2012) Sensitive Quantitative Predictions of MHC Binding Peptides and Fragment Based Peptide Vaccines from Taenia crassiceps. J Vaccines 3: 131.

14. Gomase VS, Chitlange NR (2012) Microbial Proteomics Approach for Sensitive Quantitative Predictions of MHC Binding Peptide from Taenia ovis. J Data Mining Genomics Proteomics 3: 121.

15. Sonu M, Gomase VS (2015) Prediction of antigenic epitope from D. medinensis new paradigm of synthetic vaccine development. International Conference on Recent Research Development in Environment, Social Sciences and Humanities (ICRRDESH-15). pp: 103-107.

16. Sonu M, Gomase VS (2015) Analysis of hydrophobicity and antigenic epitope prediction from D. Medinensis. International Conference on Technologies for Sustainability-Engineering, information Technology, Management and the Environment (SUSTECH-15). pp: 44-51.

17. Sonu M, Gomase VS (2015) Analysis of Hydrophobicity and Antigenicity of
Heat Shock Protein 70 from GWD. 2nd International Conference on Recent Innovations in science, Engineering and Management (ICRISEM-15). pp: 5-33.

18. (2015) National Center for Biotechnology Information Search database.

19. Acland A, Agarwala R, Barrett T, Beck J, Benson DA, et al. (2013) Database resources of the National Center for Biotechnology Information. Nucleic Acids Res 40 (Database issue): D13-25.

20. Bairoch A, Apweiler R, Wu CH, Barker WC, Boeckmann B, et al. (2005) The Universal Protein Resource (UniProt). Nucleic Acids Res 33: D154-159.

21. Hopp TP, Woods KR (1981) Prediction of protein antigenic determinants from amino acid sequences. Proc Natl Acad Sci USA 78: 3824-3828.

22. Welling GW, Weijer WJ, van der Zee R, Welling-Wester S (1985) Prediction of sequential antigenic regions in proteins. FEBS Lett 188: 215-218.

23. Parker KC, Bednarek MA, Coligan JE (1994) Scheme for ranking potential HLA-A2 binding peptides based on independent binding of individual peptide side-chains. J Immunol 152: 163-175.

24. Kolaskar AS, Tongaonkar PC (1990) A semi-empirical method for prediction of antigenic determinants on protein antigens. FEBS Lett 276: 172-174.

25. Emini EA, Hughes JV, Perlow DS, Boger J (1985) Induction of hepatitis A virusneutralizing antibody by a virus-specific synthetic peptide. J Virol 55: 836-839.

26. Karplus PA, Schulz GE (1985) Prediction of chain flexibility in proteins: a too for the selection of peptide antigen. Naturwissenschaften 72: 212-213.

27. Sweet RM, Eisenberg D (1983) Correlation of sequence hydrophobicities measures similarity in three-dimensional protein structure. J Mol Biol 171: 479488.

28. Kyte J, Doolittle RF (1982) A simple method for displaying the hydropathic character of a protein. J Mol Biol 157: 105-132.

29. Abraham DJ, Leo AJ (1987) Extension of the fragment method to calculate amino acid zwitterion and side chain partition coefficients. Proteins 2: 130-152.

30. Bull HB, Breese K (1974) Surface tension of amino acid solutions: a hydrophobicity scale of the amino acid residues. Arch Biochem Biophys 161 $665-670$.

31. Miyazawa S, Jernigen RL (1985) Estimation of Effective Interresidue Contact Energies from Protein Crystal Structures: Quasi-Chemical Approximation. Macromolecules 18: 534-552.

32. Roseman MA (1988) Hydrophilicity of polar amino acid side-chains is markedly reduced by flanking peptide bonds. J Mol Biol 200: 513-522.

33. Wilson KJ, Honegger A, Stötzel RP, Hughes GJ (1981) The behaviou of peptides on reverse-phase supports during high-pressure liquid chromatography. Biochem J 199: 31-41.

34. Cowan R, Whittaker RG (1990) Hydrophobicity indices for amino acid residues as determined by high-performance liquid chromatography. Pept Res 3: 75-80. 